\title{
Multi-focus Image Fusion Algorithm Based On Prewitt Edge Detect Information Motivated PCNN
}

\author{
Chao-ben $\mathrm{Du}^{1,2,{ }^{*}}$ and She-sheng Gao ${ }^{1}$ \\ ${ }^{1}$ School of Automatic, Northwestern Polytechnical University, Xi'an, 710072 China \\ 2 State Grid Gansu electric power company Jinchang power supply company, \\ Jinchang, 737100 , China
}

Keywords: Prewitt, PCNN, Image Fusion.

\begin{abstract}
In this paper, a novel prewitt edges operator information in spatial domain is employed as one type of feature to motivate pulse coupled neural networks (PCNN) is proposed, according to humans are often sensitive to edges and directional features. Furthermore, blocked edges information measurement is employed to reduce the computing complexity. Experimental results on subjective and objective evaluation criterion demonstrate that the Prewitt edge operator information Motivated PCNN(Prewitt-PCNN) outperforms typical SML-CT,SF-NSCT-PCNN、NSCT and SCBG fusion algorithms.
\end{abstract}

\section{Introduction}

Image Fusion is a rapidly developing research area widely used in smart transportation, remote sensing, computer vision, biomedical imaging and so on[1-2]. The purpose of image fusion is to combine different images from several sensors to create a new image, which will be more comprehensive and accurate. Furthermore, suitable for other image processing tasks a human or operator .

Pulse Coupled Neural Networks (PCNN) was developed by Eckhorn et al in 1990 ,which based on the experimental observations of synchronous pulse bursts in cat and monkey visual cortex [3].It owns some excellent characters such as pulse synchronization of neurons and the global coupling , and has been proven suitable for image processing.

Currently, PCNN has widely used in image fusion [4-5]. In spite of many multi-focus image fusion methods based on PCNN or NSCT transform. Chunhui Zhao use redundant-lifting NSWMDA and adaptive PCNN fuse Image [5]. D. Agrawal have used spatial frequency-energy of Laplacian [6] or Y. Chai have used the improved sum-modified Laplacian (SML) [7] to motivate PCNN. But it still exist problems which cannot meet our requirements. As a matter of fact, humans are often sensitive to d directional features and edges information[8]. So another important spatial-based images fusion method called the focused region-based method can detect the clear regions of input images, and then directly transferred the pixels from clear regions into the fused image [9-11]. However, these methods may generate discontinuous phenomena and artificial information at the boundaries of focused regions ,because the boundary cannot be determined accurately[12].

Based on the above analysis, an image fusion method Based On Prewitt edge detect information Motivated PCNN is proposed in this paper. Which employded prewitt edges detect information in spatial domain is employed as one type of feature to motivate pulse coupled neural networks, according to humans are often sensitive to edges and directional features. Furthermore,blocked edges information measurement is employed to reduce the computing complexity. Experimental results demonstrate that the proposed method is better than more existing PCNN-based fusion methods, in terms of both subjective and objective evaluations. 


\section{Prewitt Operator}

Prewitt operator is a first-order differential operator edge detection, which using the image pixels vertically and horizontally adjacent dot gradation difference . Reached the edge of the extremum detection edge, to remove part of the pseudo-edge, it has a certain noise smoothing effect, the basic principle is that the image of the airspace, the use of horizontal and vertical directions templates and images to complete convolution neighborhood, these two templates are used to detect horizontal directions, vertical edge[13] .In this paper we utilize novel Prewitt magnitude to detect edge features. The Prewitt operators of horizontal and vertical are shown in (1)(2), where For digital image $f(x, y)$, Prewitt operator is defined as:

$$
\begin{array}{r}
D_{x}(i, j)=[f(i+1, j-1)-f(i-1, j-1)]+[f(i+1, j)-f(i-1, j)]+[f(i+1, j+1)-f(i-1, j+1)] \\
D_{y}(i, j)=[f(i-1, j+1)-f(i-1, j-1)]+[f(i, j+1)-f(i, j-1)]+[f(i+1, j+1)-f(i+1, j-1)]
\end{array}
$$

Edge matrix obtained according to Prewitt operator as:

$$
P(i, j)=\sqrt{G_{x}(i, j)^{2}+G_{y}(i, j)^{2}}
$$

In this paper we improve on $P(i, j)$, so $P(i, j)$ is defined as:

$$
P(i, j)=\sqrt[4]{G_{x}(i, j)^{2}+G_{y}(i, j)^{2}}
$$

Blocked edges information measurement is employed to reduce the computing complexity, decompose the source images into blocks $[3 \times 3]$ and then measure the edge information in each block.

\section{PCNN-based Fusion Algorithm}

In image processing, Pulse coupled neural networks (PCNN) is a single layer pulse coupled neural cells with a two dimensional connection as shown in Fig.1. Pulse coupled neural networks (PCNN) is a feedback network and each PCNN neuron consists of four parts[14]: the external stimulus ,the receptive field, the modulation field, and the pulse generator as shown in Fig.2.

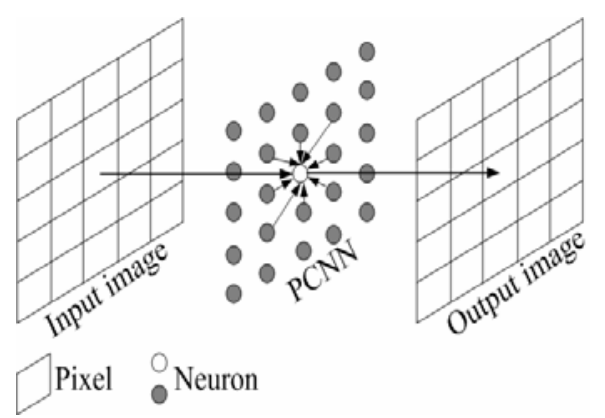

Fig.1 Connection model of PCNN neuron

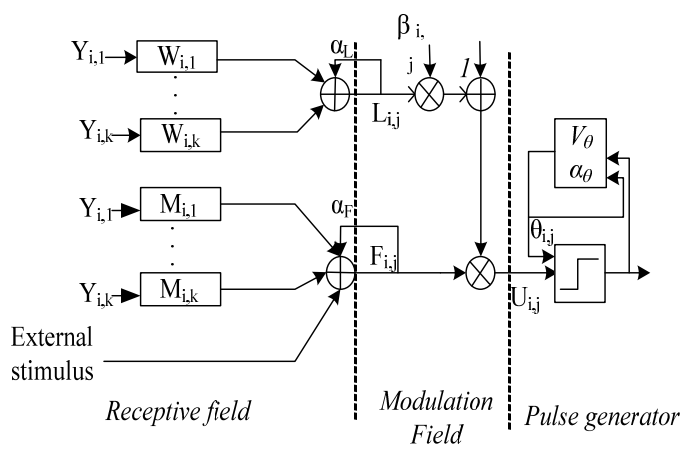

Fig .2 the Framework of PCNN

In the simplified network, the variables satisfy the following mathematic model as follows: 


$$
\left\{\begin{array}{c}
F_{i, j}^{k}=P_{i, j}^{k} \\
L_{i, j}^{k}[n]=V_{L} \sum_{p q} W_{i j, p q} Y_{p q}[n-1]+\exp \left(-\alpha_{L}\right) L_{i, j}^{k}[n-1] \\
U^{k}{ }_{i, j}[n]=F^{k}{ }_{i, j}[n]\left(\alpha+\beta L_{i, j}^{k}[n]\right) \\
\theta_{i, j}[n]=V_{\theta} Y_{i, j}{ }^{k}[n-1]+\exp \left(-\alpha_{\theta}\right) \theta^{k}{ }_{i, j}[n-1] \\
Y^{k}{ }_{i, j}[n]=\operatorname{step}\left(U_{i, j}[n]-\theta_{i, j}[n]\right) \\
X_{i, j}^{k}[n]=1 /\left(1+\exp \left(-2\left(U^{k}{ }_{i, j}-\theta^{k}{ }_{i, j}\right)\right)\right. \\
T^{k}{ }_{i, j}[n]=T^{k}{ }_{i, j}[n-1]+Y^{k}{ }_{i, j}[n]
\end{array}\right.
$$

In s (5) (6) (7) (8), the feeding input $F_{i, j}^{k}$ is equal to the normalized orientation information $P^{k}{ }_{i, j}$ in $k$-th block centred at $(i, j)$ pixel. $W_{i j}$ is the synaptic gain strength. The linking input $L_{i, j}^{k}[n]$ is equal to the sum of neurons firing times in linking range. $\alpha_{L}$ is the decay constants. $V_{\theta}$ and $V_{L} \quad$ are the amplitude gain. $U_{i, j}^{k}[n]$ is total internal activity. $\beta$ is the linking strength. $\theta_{i, j}^{k}$ is the threshold. Subscripts $p, q$ are the size of linking rang in PCNN. $n$ denotes the iteration times. If $U_{i, j}^{k}[n]$ is larger than $\theta_{i, j}^{k}$, then the neuron will generate a pulse $Y_{i, j}^{k}[n]=1$.also called one firing times. In fact, sum of $Y^{k}{ }_{i, j}[n]$ in $n$ iteration is often defined as (6) and employed to represent image information. One often analyse $T^{k}{ }_{i, j}[n]$ instead, rather than $Y_{i, j}^{k}[n] . x_{F, i j}^{k}, x_{1, i j}^{k}$ and $x_{2, i j}^{k}$ denote the pixels in the $k-t$ th block of the fused image and two source images respectively.

\section{Prewitt Edge Detect Information Motivated PCNN-Based Fusion Algorithm (Prewitt-PCNN)}

In this paper, Prewitt edge detect information introduced in section 2 is considered as one type of feature to motivate PCNN. The schematic diagram of the Prewitt-PCNN-based algorithm is shown in Fig.3.

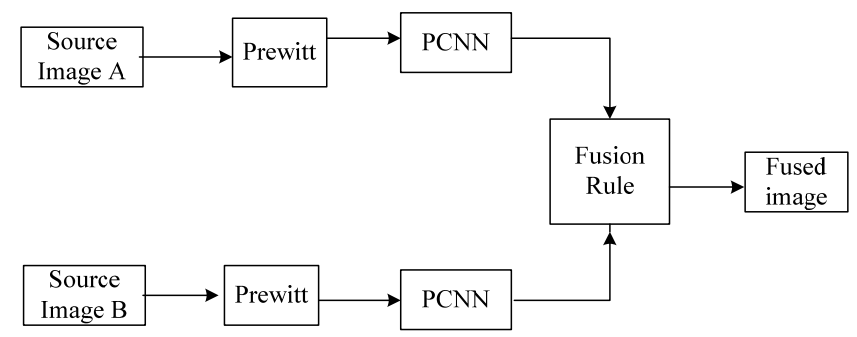

Fig.3 Schematic diagram of existed Prewitt-PCNN-based fusion algorithms

The proposed Prewitt-PCNN-based fusion algorithm is implemented as follows:

Step1: Decompose the source images into blocks ${ }^{[3 \times 3]}$ and measure the edge information of input image in each block. Then the edge information matrix $P^{A}{ }_{i, j}$ and $P^{B}{ }_{i, j}$ of the two images to be fused is calculated by using the (4) calculation.

Step2: The edge information matrix $P^{A}{ }_{i, j}$ and $P^{B}{ }_{i, j}$ of each source image is input to PCNN and pulse of neurons generated according to (5). Then sum of firing times $T_{i, j}^{k}[n]$ is calculated as (6).

Step3: Get the decision map $D_{i j}^{k}$ based on (6), which is the fusion rule proposed in this paper. which is block of source images with larger edge information is employed as the blocks of the fused images. 
Step4: According to the filtered decision map $D_{i j}^{k}$ by using (7) and (8) reconstruct the fused image $x_{F, i j}^{k}$.

$$
\begin{gathered}
D_{F, i j}^{k}=\left\{\begin{array}{l}
1, i f: T_{1, i j}^{k}(\mathrm{n}) \geq T_{2, i j}^{k}(\mathrm{n}) \\
0, i f: T_{1, i j}^{k}(\mathrm{n})<T_{2, i j}^{k}(\mathrm{n})
\end{array}\right. \\
X_{F, i j}^{k}=\left\{\begin{array}{l}
x_{1, i j}^{k}, \text { if }: D_{i j}^{k}=1 \\
x_{2, i j}^{k}, \text { if }: D_{i j}^{k}=0
\end{array}\right.
\end{gathered}
$$

\section{Experimental Results and Evaluation}

In order to show the advantages of the new image fusion method, using clock and pepsi selected as test images to verify the algorithm proposed in this paper.Fig.4 (a) are (b) are source Multi-focus images with size $512 \times 512$. In the experiments, parameters of PCNN set as

$$
\begin{aligned}
& p \times q=3 \times 3, \alpha_{L}=0.06931, \alpha=1, \alpha_{\theta}=0.2, V_{\theta}=20, V_{L}=1, \beta=0.5, \\
& W=[0.707,1,0.707 ; 1,0,1 ; 0.707,1,0.707]
\end{aligned}
$$

Table 1 Comparison on objective criteria of different methods and proposed method

\begin{tabular}{ccccccc}
\hline images & evaluation & SML-CT & SF-NSCT-PCNN & NSCT & SCBG & proposed method \\
& $\mathrm{MI}$ & 6.5603 & 7.4051 & 8.1904 & 8.4652 & 8.5791 \\
clock & $\mathrm{Q}^{\mathrm{AB} / \mathrm{F}}$ & 0.6672 & 0.6863 & 0.7366 & 0.5946 & 0.7263 \\
pepsi & $\mathrm{MI}$ & 6.6514 & 7.7323 & 7.7230 & 8.6527 & 8.7496 \\
& $\mathrm{Q}^{\mathrm{AB} / \mathrm{F}}$ & 0.7636 & 0.7767 & 0.7258 & 0.6258 & 0.7875 \\
\hline
\end{tabular}

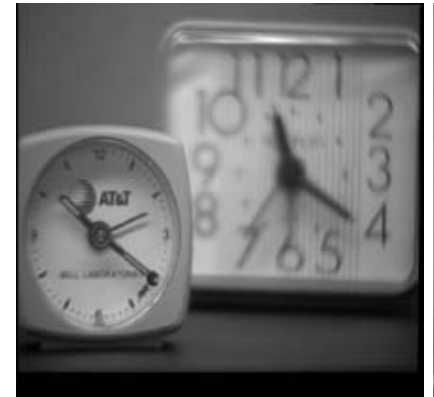

(a)

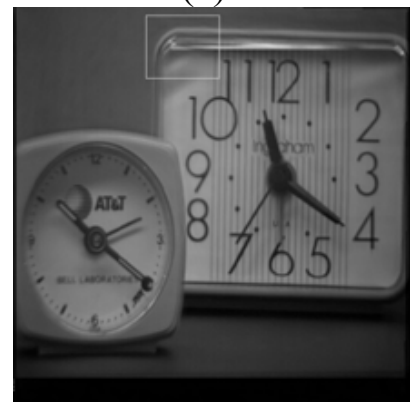

(e)

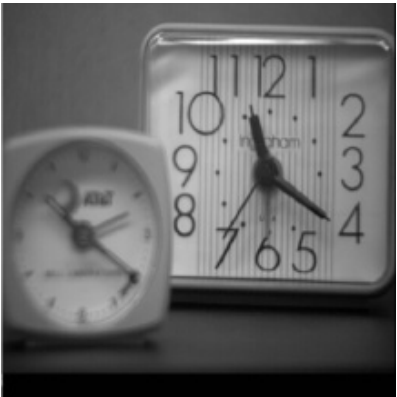

(b)

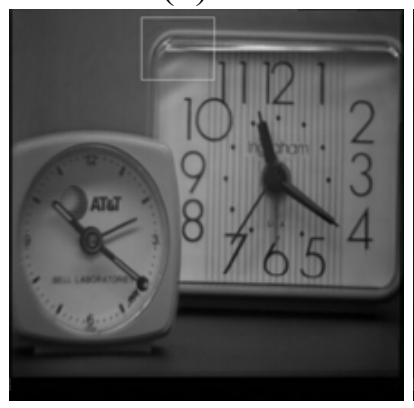

(f)

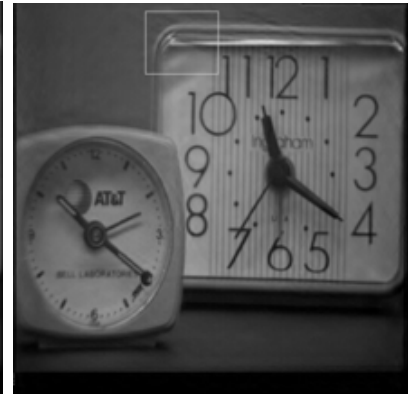

(c)

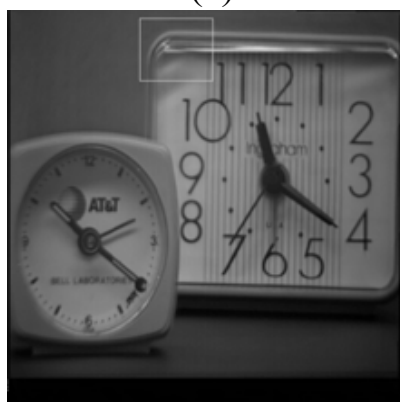

(g)

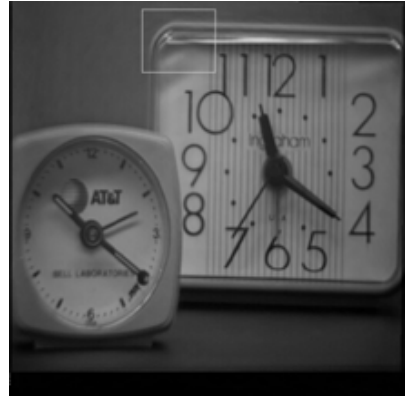

(d)

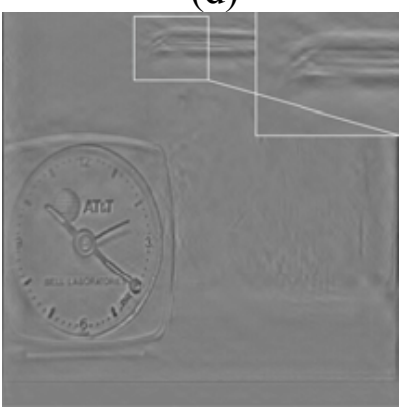

(h) 


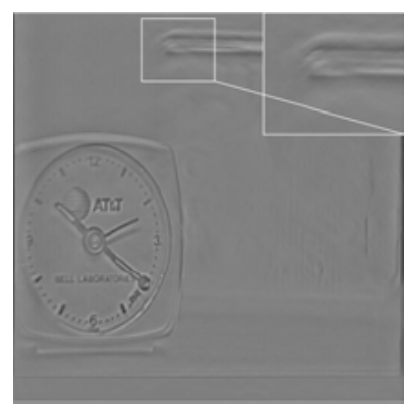

(i)

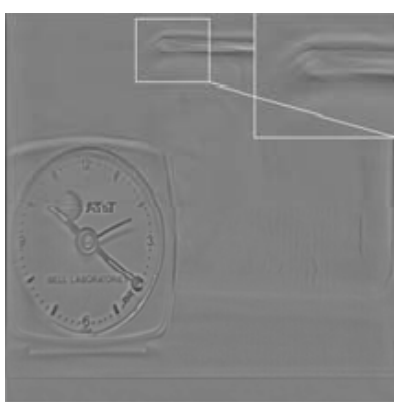

(j)

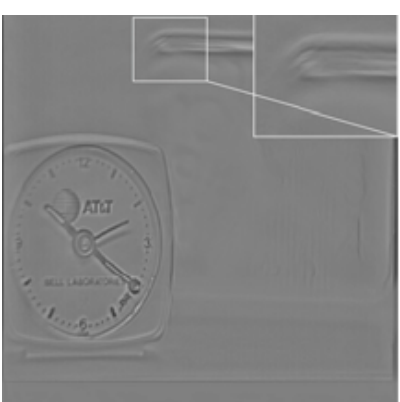

$(\mathrm{k})$

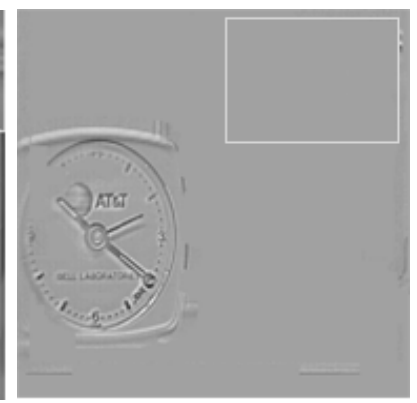

(1)

Fig.4 (a)and(b)is source image,(c), (d), (e),(f) and (g) are fused results using SML-CT, SF-NSCT-PCNN, NSCT, SCBG and proposed method respectively. (h), (i), (j),(k) and(l) are the difference image which (c), (d), (e),(f) and (g) minus the source image shown in Fig.4 (b).

Comparison of SML-CT , SF-PCNN-NSCT[15] ,NSCT[16], SCBG[17] and the proposed method in terms of both subjective and objective evaluations. Mutual information (MI) and QAB/F are employed as information-based objective criteria are used to compare the fusion results. The values of MI and QAB/F of the two sets of images are listed in Table 1. From Table 1, we can see that the values of $\mathrm{MI}$ and $\mathrm{QAB} / \mathrm{F}$ of the proposed method are all most the largest and optimal in the six fusion methods, besides the $\mathrm{QAB} / \mathrm{F}$ of the proposed method is lower than NSCT when fused 'clock' .This indicate that the fused image obtained by the proposed method can retain much more focused information from the source images.

For further comparison ,besides objective criteria, visual observation are used to compare the fusion results.Fig.4 (c)- Fig.4 (g)shows the visual observation of fused images shown in Fig.4 (a) and Fig.4 (b). Because source image is clear in the labeled part, so the lower residue features in the difference images, which are gotten by subtracting Fig.3 (a), means the better the transfer features of source images to fused images. Especially, blurry edges, presented in the zoomed out parts, show the pseudo-Gibbs phenomena which reduces visual quality of the fused images. Fig.4(l) mark portion is substantially no residual image characteristic value, in comparison with Fig.4(h)-Fig.4 (k). This shows that information of source images are best transferred into fused image in our image fusion algorithm.

\section{Conclusion and Discussion}

A novel prewitt edges operator information in spatial domain is employed as one type of feature to motivate pulse coupled neural networks (PCNN) is proposed in this paper, according to humans are often sensitive to edges and directional features. Furthermore, blocked edges information measurement is employed to reduce the computing complexity. Experimental results on subjective and objective evaluation criterion demonstrate that the Prewitt edge operator information Motivated PCNN(Prewitt-PCNN) can efficiently transfer the information from source images to fused image.

\section{Acknowledgments}

The work of this paper was supported by the National Natural Science Foundation of China (Proj ect Number: 61174193) and the Specialized Research Fund for the Doctoral Program of Higher Edu cation (Project Number: 20136102110036).

\section{References}

[1] Ghassemian, Hassan "A review of remote sensing image fusion methods" INFORMATION FUSION, vol. 60, pp. 75-89, Nov. 2016.

[2] Ma, Yong; Chen, Jun; Chen, Chen; "Infrared and visible image fusion using total variation model," NEUROCOMPUTING, vol. 202, pp. 12-19, Aug 2016. 
[3] Eckhorn R., Reitboeck H., Arndt M., et al, "Feature linking via synchronization among distributed assemblies: Simulations of results from cat visual cortex," Neural Comp., vol.2, no.3, pp.293-307, 1990.

[4] Baochang Xu, Zhe Chen, "A multisensor image fusion algorithm based on PCNN," Fifth World Congress on Intelligent Control and Automation-WCICA 2004, Vol.4, pp. 3679-3682, 2004 .

[5]Chunhui Zhao, Guofeng Shao, Lijuan Ma, Xi Zhang Image fusion algorithm based on redundant-lifting NSWMDAand adaptive PCNN. Optik 125 (2014) 6247-6255

[6] D. Agrawal, J. Singhai, Multifocus image fusion using modified pulse coupledneural network for improved image quality, Image Process. IET 4 (6) (2010)443-451.

[7] Y. Chai, H.F. Li, M.Y. Guo, Multifocus image fusion scheme based on featuresof multiscale products and PCNN in lifting stationary wavelet domain, Opt.Commun. 284 (5) (2010) 1146-1158.

[8] Xiaobo Qu, Changwei Hu, Jingwen Yan, Image Fusion Algorithm Based On Orientation Information Motivated Pulse Coupled Neural Networks, The seventh World Congress on Intelligent Control and Automation-WCICA2008-Chongqing, China,25-27 June 2008, pp.2437-2441

[9] G. Piella, "A general framework for multiresolution image fusion: From pixels to regions," Inf. Fusion, vol. 4, no. 4, pp. 259-280, Dec. 2003.

[10] S. Li, X. Kang, J. Hu, and B. Yang, "Image matting for fusion of multi-focus images in dynamic scenes," Inf. Fusion, vol. 14, no. 2, pp. 147-162, Apr. 2013.

[11] Y. Liu, J. Jin, Q. Wang, Y. Shen, and X. Dong, "Region level basedmulti-focus image fusion using quaternion wavelet and normalized cut,"Signal Process., vol. 97, pp. 9-30, Apr. 2014.

[12] Yong Yang, Song Tong, Shuying Huang, and Pan Lin. Multi-focus Image Fusion Based on NSCT and Focused Area Detection. IEEE SENSORS JOURNAL, VOL. 15, pp.2824-2838.NO. 5, MAY 2015.

[13] Hu Zhang, Qiuping Zhu, Cien Fan, Dexiang Deng. Image quality assessment based on Prewitt magnitude[J]. Int. J. Electron. Commun. (AEU) 67 (2013) 799-803.

[14] Johnson J.L., Padgett M.L., "PCNN models and applications," IEEE Trans.Neural Networks, vol.10, no.3, pp.480-498, 1999.

[15] Xiaobo Qu, Jingwen YAN, Hongzhi XIAO, Ziqian ZHU.Image Fusion Algorithm Based on Spatial Frequency Motivated Pulse Coupled Neural Networks in Nonsubsampled Contourlet Transform Domain[J].Acta Automatica Sinica.2008, 34(12):1508-1514.

[16] Y. Chai, H. Li, and X. Zhang, "Multifocus image fusion based on features contrast of multiscale products in nonsubsampled contourlet transform domain," OPTIK. J. Light Electron Opt., vol. 123, no. 7,pp. 569-581, Apr. 2012.

[17] J. Tian, L. Chen, L. Ma, and W. Yu, "Multi-focus image fusion using a bilateral gradient-based sharpness criterion," Opt. Commun., vol. 284,no. 1, pp. 80-87, Jan. 2011. 